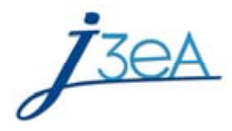

Journal sur l'enseignement des sciences et technologies de l'information et des systèmes

Dossier spécial : Formations internationales

\title{
D2I : DUT Informatique International
}

\author{
Jean-Michel Bruel et Michèle Verdier \\ Université de Toulouse, IUT de Blagnac \\ 1, pl. Georges Brassens, 31703 Blagnac Cedex, France
}

\begin{abstract}
RESUME : Nous relatons dans cet article l'expérience innovante réalisée cette année (2010/2011) d'ouverture, en $2^{\text {ème }}$ année de DUT Informatique, d'un semestre d'enseignement en langue anglaise. Les enseignements en anglais ne concernent pas toute la promotion et ne concernent pas non plus l'ensemble des enseignements de seconde année de DUT, mais permet à un groupe entier d'étudiants d'être immergés en anglais dans leur contexte de travail. Nous détaillons les objectifs et les enjeux, nous décrivons le dispositif mis en place et relatons les premiers résultats dont certains n’étaient pas attendus.
\end{abstract}

Mots clés : dispositif pédagogique, immersion, anglophone, DUT, S3-S4, informatique.

\section{INTRODUCTION}

Cet article vise à rapporter l'expérience de la mise en place d'une véritable immersion en anglais d'une partie de nos étudiants de DUT $2^{\text {ème }}$ année. Cette expérience, inédite à notre connaissance au niveau $\mathrm{Bac}+2$ par sa durée et le nombre de modules concernés, a été mise en place à la rentrée 2010. Nous relatons dans cet article l'origine et les raisons de cette mise en place, les difficultés que nous rencontrons et les bénéfices déjà observés pour les étudiants concernés. La section 1 décrit le contexte et l'origine de cette expérience. La section 2 décrit les raisons qui nous ont poussés à nous lancer dans cette aventure. La section 3 décrit sous une forme synthétique la fiche signalétique décrivant l'expérience. La section 4 décrit les premiers retours de cette jeune expérience, et enfin la section 4 conclut sur le devenir de cette expérience.

\section{POURQUOI UN DUT « INTERNATIONAL »}

Le domaine de l'informatique est principalement piloté par le monde anglophone. Le vocabulaire est massivement d'origine anglaise, les langages de programmation utilisent des instructions anglaises, les premières documentations accessibles pour un logiciel récent ne sont souvent disponibles qu'en anglais dans un premier temps, etc. L'anglais a donc depuis toujours une place importante dans les cursus informatiques, et plus particulièrement dans les cursus technologiques comme celui du DUT Informatique. Le Programme Pédagogique National (PPN, cf. [2]), qui régit nos curriculums, mentionne un volume d'environ $140 \mathrm{~h}$ d'anglais. De même, dans les pratiques pédagogiques plus techniques, une utilisation de l'anglais est mise en avant. Par exemple, dans les cours de Systèmes d'Exploitation, les étudiants sont invités à utiliser (et parfois interrogés en contrôle sur) les «man » (manuels, ou explications) des commandes vues en travaux pratiques. Cela reflète en effet la majorité des environnements de travail que nos étudiants vont rencontrer dans leur carrière d'informaticien. Les étudiants qui choisissent la filière DUT sont au fait de cette impor- tance de l'anglais qui intervient même dans le recrutement des étudiants en ce qui concerne notre département. Certains d'entre eux sont un peu réticents, mais tous admettent la nécessité d'un minimum de connaissance et de pratique. Et certains réclament même plus d'expérience. Ils sont de toute façon baignés dans un univers anglophone (séries télévisées en streaming pour ne pas attendre la sortie en France, jeux en réseaux avec des " partenaires » de toute la planète, etc.).

En parallèle, et plus particulièrement dans le contexte industriel toulousain fortement empreint d'aéronautique et de mobilité professionnelle, nous commençons à voir de plus en plus de propositions de stage (ou d'embauche) portant des mentions explicites de maîtrise de l'anglais, voire de contexte complètement international (encadrant, réunions, etc.).

Enfin, troisième constat, de nombreux étudiants de nos promotions partent à l'étranger : la majorité dans le cadre de leur stage, certains peuvent partir pour un semestre Erasmus, d'autres dans le cadre de la poursuite d'étude. Mais notre département n'accueille aucun étudiant Erasmus, en tout cas pas sur le dernier quadriennal. Après discussions avec les collègues des relations internationales et des autres départements, nous avons fait le constat que la barrière de la langue française pour effectuer des études technologiques difficiles (le programme de DUT est le double en volume horaire d'un parcours classique de licence) était un obstacle à la venue d'étudiants étrangers dans nos formations. Or, toutes les formations qui accueillent des étrangers le disent, la richesse du brassage engendrée dans les promotions par ces étudiants étrangers, l'ouverture au monde et l'ouverture d'esprit que ces situations véhiculent est un atout pour toute la promotion. Forts de ces constats, il est apparut évident pour nous que proposer de réaliser notre formation en anglais pouvait être une solution idéale pour les faire converger vers une solution intéressante. L’idée de ce projet étant d'atteindre à terme un véritable label «DUT Informatique International », nous avons baptisé ce projet le D2I (du fait de l'acronyme DII formé par le Dut Informatique International, mais aussi en clin d'œil à un acronyme très à la mode à l'université, 
le C2I. Enfin, le choix de mener l'expérience en DUT plutôt qu'en Licence pro a été guidé par l'attractivité et le caractère novateur de notre initiative, ainsi que des considérations pratiques d'organisation. La section suivante décrit comment nous nous y sommes pris pour arriver à lancer l’expérience.

\section{DESCRIPTION DE L'EXPERIENCE}

\subsection{Contexte}

Le département d'Informatique de l'IUT de Blagnac est un département de taille assez standard (4 groupes de 26 étudiants en $1^{\text {ère }}$ année et 3 groupes en $2^{\text {ème }}$ année). L’équipe pédagogique est constituée de 17 permanents dont 10 enseignants-chercheurs (ayant donc une pratique scientifique de l'anglais). L'équipe pédagogique est particulièrement dynamique et soudée. Ceci est un point important car l'adhésion de l'équipe pédagogique au projet est un ingrédient indispensable pour sa mise en place et sa réussite. Le département compte une permanente certifiée d'anglais qui fait également intervenir des vacataires en anglais (vu le volume d'enseignement à assurer). Les raisons ont été évoquées à la section précédente et pour ce qui concerne le contexte, il est bon de rajouter que, comme souvent dans ce genre d'expérimentation, les conditions financières était simples : le coût zéro ! Nous avons la chance d'être rattaché à une université de Sciences Humaines et Sociales, sensible aux initiatives pédagogiques, et aux langues étrangères en particulier, et d'avoir également une équipe de direction ouverte et compréhensive. Tous les ingrédients étaient donc réunis pour se lancer : des porteurs de projets motivés, une équipe pédagogique prête à tenter l'expérience, et une administration favorable. Restait à trouver les moyens de se lancer avec les contraintes budgétaires de ne pas entraîner de surcoût. Nous détaillons les aspects organisationnels dans la section 3.8.

\subsection{Recrutement}

Nous avons décidé en fin d’année 2009/2010 de démarrer l'expérience. Il n'a donc pas été possible de faire la promotion du «D2I ». Le recrutement a donc consisté pour cette année à ouvrir le groupe de TD de 26 étudiants à nos étudiants s'inscrivant en $2^{\text {ème }}$ année de DUT Informatique à la rentrée.

En effet, une des difficultés administratives qui nous inquiétait concernait le fait que l'on ne peut pas imposer des cours en anglais à des étudiants français (qui ont payé pour recevoir une formation dans leur langue maternelle a priori !). Partant de l'idée de constituer un groupe de Travaux Dirigés anglophone sur les 3 groupes que compte la $2^{\text {ème }}$ année de notre DUT, il fallait donc que la constitution du groupe se fasse uniquement sur la base du volontariat. Heureusement, pour cette rentrée 2010 nous avions plus de la moitié de la promotion de $2^{\text {ème }}$ année qui s'est portée volontaire (soit près de 35 étudiants pour les 26 places disponibles dans le groupe).
Sinon dans l'absolu, le D2I est accessible à tout étudiant anglophone possédant l'équivalent d'un Bac+1 en informatique (première année de B.Sc. par exemple). Mais nous revenons sur cette question en section 3.7.

\subsection{Langues d'enseignement}

Comme indiqué précédemment et comme il sera détaillé dans la partie organisation (cf. section 3.8), les cours d'amphi ont été dispensés en français aux étudiants tandis que les Travaux Dirigés ou les Travaux Pratiques étaient enseignés en anglais. Parmi l'ensemble des disciplines enseignées en $2^{\text {ème }}$ année, seule une partie a été enseignée en anglais. En effet pour des raisons de commodité et pour le lancement de l'expérience, et vu l'absence de purs anglophones, certaines matières, déjà complexes pour nos étudiants d'informatique (comme une partie des mathématiques, l'économie gestion) n’ont pas été enseignées en anglais. Néanmoins, même dans ces matières (et les étudiants étant demandeurs), des supports en anglais ou en tout cas des éléments de vocabulaire anglais ont été distribué aux étudiants. Le coût (marginal) de ces traductions n’a pas été évalué. Il est a noté qu’à une ou deux exceptions près, aucun intervenant n'était anglophone, mais tous étaient volontaires.

\subsection{Mobilités réelles ou virtuelles des étudiants}

Notre département s’investit pour la mobilité de nos étudiants à l'international, mais pas spécifiquement dans le cadre de l'expérience du D2I relatée ici. Nous ne ferons donc que mentionner ici qu'une part significative des étudiants qui vont partir dans le cadre d'échanges ou de stage pour cette année universitaire est issue du groupe anglophone. Ce sont en effet d'une part les étudiants en général les plus motivés pour les études à l'étranger, mais d'autre part, la sélection aux places se faisant en partie sur le niveau d'anglais, il n'est pas surprenant que les étudiants du groupe anglophone aient été classés plus souvent en tête des candidatures.

\subsection{Mobilités réelles ou virtuelles des enseignants}

Comme indiqué précédemment, le projet D2I ne comporte pas pour l'instant de volet collaboration. Il n'empêche que nous avons un certain nombre de collaborations avec des universités étrangères (ITTallaght Dublin, Universidad Politecnica de Valencia etc.). Nous avons profité d'échanges Erasmus d'enseignants (Andrew Donnellan, ...) pour faire intervenir ces anglophones dans la formation.

\subsection{Reconnaissance « internationale »}

La seule reconnaissance actuelle à l'international de notre diplôme concerne une entente signée et validée par le ministère entre l'université de Chicoutimi au Québec et l'Assemblée des Chefs de 
Département Informatique (ACDI). Cette entente permet à nos DUT Informatique de poursuivre en B.Sc. $(\mathrm{Bac}+3)$ dans deux spécialités (dont une prisée de « jeux vidéo »). Les étudiants bénéficient de plus de droits d'inscriptions privilégiés. De plus, le B.Sc. nordaméricain étant légèrement plus conséquent que nos licences L3, les étudiants rejoignent sans soucis des Masters à leur retour en France.

Pour ce qui concerne le D2I, il ne s'agit pour l'instant pas d'une formation réellement internationale. Elle n'est pour l'instant qu'anglophone et seul un nombre conséquent d'inscriptions d'étudiants étrangers pourra nous donner véritablement ce label. La prochaine étape vers cette reconnaissance consiste tout de même à faire la démonstration que $70 \%$ de nos enseignements se font en anglais ce qui nous permettra d'être inscrits dans la liste des formations françaises « internationales » du système de promotion Campus France dont le but est de permettre de centraliser les candidatures d'étudiants étrangers dans les filières françaises.

\subsection{Etudiants concernés}

Nous avons la possibilité d’accueillir 26 étudiants (un groupe) en 2ème année de DUT Informatique. Le public concerné est donc celui des étudiants ayant déjà validé une année dans leur licence ou B.Sc. local, et souhaitant passer soit le $1^{\text {er }}$ semestre de la $2^{\text {ème }}$ année de DUT, soit l'année complète dans notre formation. La deuxième année de DUT consiste en un premier semestre classique de 450 heures. Le deuxième semestre est en fait un demi-semestre (8 semaines seulement) puisque les étudiants partent en stage pour 10 ou 12 semaines. Le contexte aéronautique toulousain est propice à ce que les étudiants anglophones décrochent un stage sur place. Ils peuvent aussi envisager retourner faire ce stage dans leur pays. La difficulté de recrutement vient du fait que le système des IUT est assez éloigné des études universitaires classiques (il ne rentre pas vraiment dans le «moule» L/M/D par exemple). Il n'est donc pas évident de communiquer sur les spécificités et les intérêts pour les étudiants à participer à nos formations. Quelques pistes sont néanmoins possible car de plus en plus de pays (Allemagne en tête) misent sur l'apprentissage et des formations similaires au système IUT voient le jour et peuvent peut-être constituer un vivier intéressant de candidats potentiels.

\subsection{Organisation}

En 2ème année nous avons 3 groupes de TD. Etant donné la contrainte évoquée plus haut de fonctionner à coût constant, la solution la plus simple et la plus économique a consisté pour nous à décider que pour l'un de ces 3 groupes, les enseignements aient lieu en anglais. Les étudiants sont donc en promotion complète pour les cours en amphi (15\% du temps environ) et suivent alors des cours en français. Pour la constitution du groupe il a été fait appel à des volontaires. Il est assez logique de trouver une certaine corrélation entre le niveau général des étudiants et leur motivation pour le groupe anglophone (cf. Fig.1). Nous avons essayé de ne pas faire de ce groupe un groupe " élitiste ». Le niveau d'anglais a donc été prépondérant dans notre choix. Les résultats du $1^{\mathrm{er}}$ semestre de la $2^{\text {ème }}$ année (cf. Fig.2) permettent d'être satisfait car ils montrent que ce groupe s'est comporté comme les autres en terme de résultats. Un excès de réussite aurait pu entrainer une stigmatisation de ce groupe vu comme une élite, et de mauvais résultats auraient pu entrainer l'arrêt de l'expérience (une moindre moyenne aurait été interprétée comme le résultat de difficultés liées à l'anglais).

\section{RETOUR D'EXPERIENCE}

Il est un peu tôt pour faire un réel bilan de cette première année d'expérience. Néanmoins, un certain nombre de points peuvent être notés.

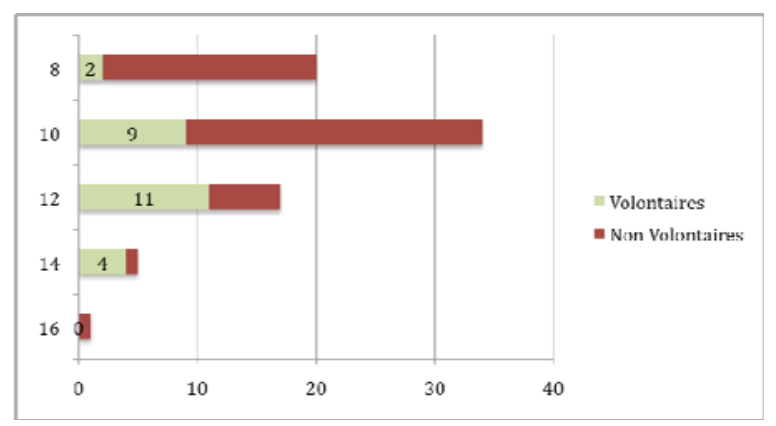

Fig. 1 : Répartition du nombre de candidats selon leur moyenne générale à l'issue de la $1^{\text {ère }}$ année

Un des effets les plus bénéfiques de cette expérience, et que nous souhaitons mettre en avant, concerne la participation des étudiants. Une des inquiétudes concernant cette expérience concernait le fait que la participation des étudiants pouvait être impactée par la barrière de la langue. Bien que tous volontaires, les étudiants allaient-ils poser autant de questions dans les modules anglophones que les autres? Le fait de leur demander de s'exprimer en anglais n'allait-il pas les bloquer ? De manière étonnante il s'est produit au contraire un surcroît de participation. Plusieurs explications peuvent être avancées :

- les étudiants étaient là pour participer et un petit noyau maîtrisait déjà bien l'anglais,

- il s'est produit une sorte d'émulation à voir les camarades se prêter au jeu («pourquoi pas moi ?»),

- de l'aveu même des étudiants, le fait de voir les enseignants parler un anglais pas forcément parfait, tout en se faisant comprendre, et surtout sans en être ni gênés ni même troublés, a été un véritable déclencheur pour eux, 
- nous ne nous intéressions pas du tout au niveau d'anglais. L'essentiel était de se faire comprendre. Certaines séances de brainstorming pour trouver la bonne traduction d'une phrase étaient même sources de jeux anglophones fort intéressantes.

Il va de soi que l'expérience n’a pas été un succès à $100 \%$. Quelques modules ont opéré un retour au français. Soit parce que le module était vraiment complexe en anglais pour les étudiants (le nombre des « breaks » d'explications françaises est alors devenu dominant). Soit que l'enseignant trouvait trop contraignant de parler anglais alors qu'il n'y avait pas un seul « non francophone » parmi les étudiants.

Un autre point fort intéressant pour ceux qui voudraient tenter l'expérience dans leur formation concerne l'ensemble des circuits administratifs. En effet après avoir annoncé notre intention de mener cette expérience anglophone sur une échelle jamais encore proposée, nous n'avons reçu aucune objection, que ce soit de la direction de l'IUT ou de l'Université. C'est un point important pour la motivation de l'équipe enseignante. Nous aurions aimé obtenir plus de soutien des services des relations internationales (RI) de l'Université ou même du Pôle de Recherche et d'Enseignement Supérieur (PRES) de Toulouse. Il a été en effet très dur d'obtenir un minimum d'attention. Aucune publicité n’a été faite en interne à l'université ni même au PRES malgré l'information que nous leur avons transmise, comme par exemple la traduction anglaise du Programme Pédagogique National qui régit notre DUT informatique.

Une de nos idées fortes de départ était de lutter contre le fait que les Erasmus ne viennent pas en France à cause de la langue. Il est trop tôt pour savoir si cet objectif est atteint. En effet, notre «D2I » n'en étant qu’à sa première année, nous espérons que la publicité faite cette année autour de cette opportunité pour des étudiants de niveau $\mathrm{Bac}+1$ de venir étudier en France et d'obtenir un diplôme français reconnu portera ses fruits pour la rentrée 2011. Il s’agit en effet d'une condition indispensable pour pouvoir poursuivre l'expérience, comme nous en discuterons dans la section suivante.

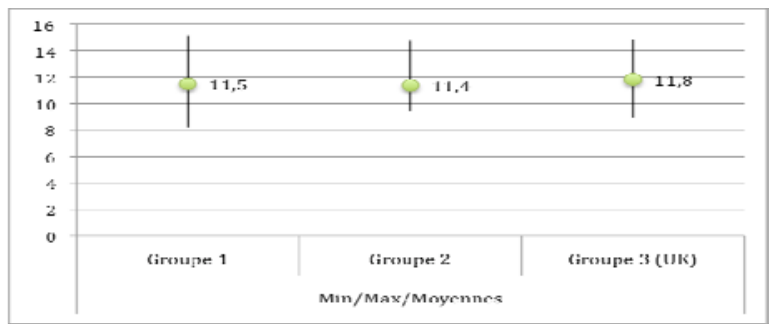

Fig. 2 : Réussite comparative des 3 groupes de $2^{\text {ème }}$ année.

Un retour intéressant de la part des étudiants concerne les enseignants anglophones (cf. section 3.5). Bien que plus difficiles à suivre pour les étudiants français, ces enseignants ont été plus appréciés que les francophones parlant anglais. La raison est surprenante : le fait de savoir que l'enseignant ne parle pas français, et que donc il ne va non seulement pas se mettre à donner des explications complémentaires en français, mais en plus qu'il ne va pas pouvoir répondre à des questions formulées autrement qu'en anglais (même approximatif), a entraîné chez les étudiants une bien plus grande concentration. Ils nous ont avoués être même bien plus concentrés qu'avec leurs enseignements en français. En effet, quelques minutes d'inattention suffisent à être vraiment perdu avec un discours en langue étrangère. Ils sont donc sortis fatigués de ce genre de cours, mais admettent que la concentration leur a permis de mieux appréhender le contenu de la matière enseignée. Ils étaient même demandeurs pour renouveler plus souvent l'expérience.

\section{CONCLUSION}

Sans financement particulier (il s'agissait d'une condition initiale pour lancer l'expérience), et avec la bonne volonté d'un département dynamique et volontaire, nous avons réussi à donner à un petit groupe d'étudiants français un petit plus sur leur CV en donnant un caractère « anglophone » à une partie de leur cursus. Pour pouvoir prétendre au titre de DUT Informatique International, il nous faut absolument passer à l'étape suivante à savoir accueillir une majorité d'étudiants étrangers_dans ce groupe. Il en va de la crédibilité de cette option, de son écho auprès des formations étrangères. C'est également important pour la motivation et l'implication de nos collègues enseignants qui se sont prêtés à l'exercice périlleux d'enseigner dans une langue qui ne leur est pas maternelle. Enseigner en anglais, même quand on aime ça ou que la discipline s'y prête, est une chose. Mais faire la même chose en sachant pertinemment que tout l'auditoire est francophone demande une dose supplémentaire de courage. Il est en effet très tentant sur des parties un peu complexes, de «switcher » en français pour un instant d'explications plus claires. Même une minorité de non francophones suffit à interdire cette éventualité et permet à l'enseignant volontaire de se rassurer quant à son obligation de parler anglais. Sans même parler de l'aide précieuse, à la fois pour ses camarades et pour l'enseignant, que peut apporter un « native speaker» dans un groupe. Nous allons donc maintenant faire porter nos efforts sur la publicité à l'étranger de notre filière en espérant voir les candidatures augmenter d'année en année et nous espérons que cette publication y contribuera d'une certaine façon.

\section{REMERCIEMENTS}

Les auteurs tiennent à remercier chaleureusement leurs collègues du département informatique pour leur implication dans cette expérience, ainsi que les étudiants volontaires pour avoir essuyé les plâtres d'une organisation balbutiante avec bonne humeur et bonne intelligence. 


\section{Bibliographie}

[1] “National Teaching Program”, traduction du Programme Pédagogique National (PPN) en anglais. Disponible à l’URL : http://tinyurl.com/6dj95em

[2] http://www.iut-informatique.fr/en_presentation.php, Site anglophone des IUT Informatique de France. 